\section{CANCER METABOLISM}

\section{A-FABP links obesity and breast cancer}

Obesity is known to increase the risk of developing certain cancers, such as breast cancer and oesophageal cancer; however, the mechanisms underlying this association have been unclear. Now, new research conducted by Bing Li and colleagues indicates that increased levels of adipose fatty acid binding protein (A-FABP) are associated with an increased risk of breast cancer.

"As the two most predominant populations in breast cancer stroma, macrophages and adipocytes have central roles in tumorigenesis and progression of breast cancer; A-FABP attracted our attention due to its abundant expression in both macrophages and adipocytes," explains Li. By analysing serum samples from women with obesity before and after they underwent Roux-en-Y gastric bypass surgery, the researchers established that circulating levels of A-FABP were increased in conditions of obesity and that levels reduced as BMI declined.

To investigate the link between A-FABP and increased risk of breast cancer, Li and colleagues collected serum samples from 285 women with breast lesions before they underwent diagnostic biopsies. Importantly, they found that circulating levels of A-FABP were elevated in women with obesity who were diagnosed with breast cancer, but not in lean women who received this diagnosis. In addition, experiments in mice fed a high-fat diet to induce obesity showed that ablation of A-FABP did not affect the degree of obesity, but did inhibit tumour growth and development in the mammary tissue. In vitro, $\mathrm{Li}$ and colleagues showed that A-FABP treatment resulted in activation of the IL-6-STAT3-ALDH1 signalling pathway and an increase in levels of STAT3-activating cytokines, which increased the stemness and aggressiveness of tumours. "Our research indicates that A-FABP is a new player in breast cancer development," says Li.

The researchers are expanding their studies in this area, including analyses of whether A-FABP expression in particular subsets of cells are associated with the development and progression of breast cancer. "We believe that these studies will facilitate its potential application in breast cancer diagnosis and immunotherapy in clinical medicine," concludes Li.

Claire Greenhill

ORIGINAL ARTICLE Hao, J. et al. Circulating adipose fatty acid binding protein is a new link underlying obesity-associated breast/mammary tumor development. Cell Metab. https://doi.org/10.1016/j.cmet.2018.07.006 (2018)

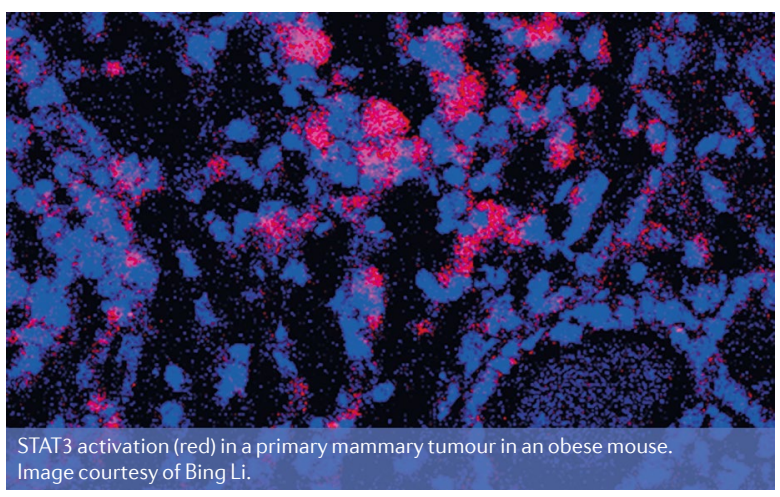

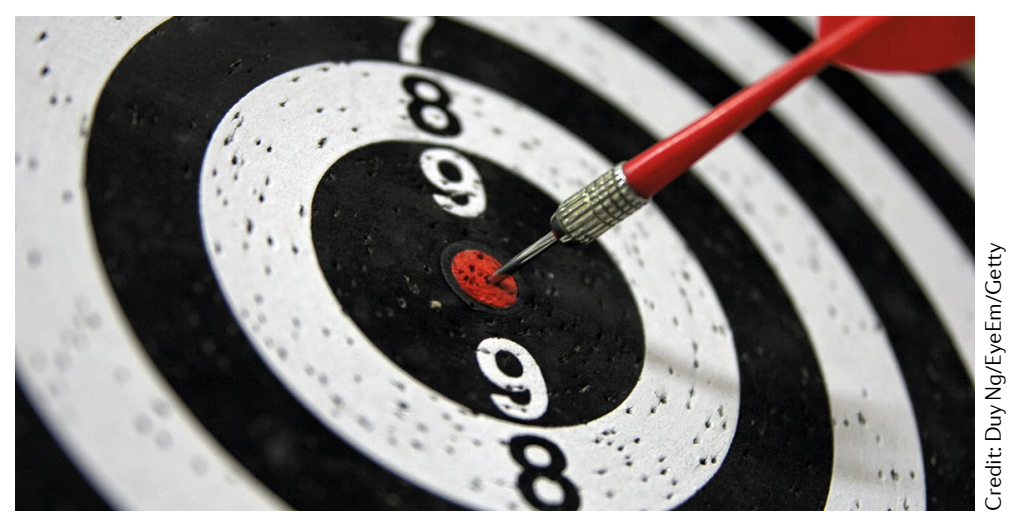

DIABETES

\title{
Epsins as a target for wound-healing therapeutics
}

A complication of type 2 diabetes mellitus (T2DM) is impaired wound healing. In patients with T2DM, impaired wound healing can also manifest as secondary lymphoedema, which is localized fluid retention and tissue swelling resulting from a compromised lymphatic system.

The molecular mechanisms that underpin impaired lymphangiogenesis in the context of T2DM are poorly understood. Now, Hong Chen and colleagues report that epsin deficiency can rescue impaired lymphatic function in a mouse model of diabetes mellitus by limiting VEGFR3 degradation.

Chen and colleagues previously demonstrated that the loss of epsins 1 and 2 in lymphatic endothelial cells prevented VEGFC-induced VEGFR3 endocytosis and degradation. "Studies have shown that diabetic hyperglycaemia hinders VEGFR3 signalling-dependent lymphangiogenesis; therefore, and given that epsins have a critical role in downregulating VEGFR3 signalling, we postulated that epsin deficiency could rescue impairment of lymphangiogenesis by preventing the degradation of VEGFR3 in diabetes mellitus."

In the present study, the authors fed inducible lymphatic-specific deficiency of epsins 1 and 2 (LEC-iDKO) mice a high-fat diet and treated them with streptozotocin (to investigate the role of epsin loss in lymphangiogenesis during T2DM). To investigate whether lymphaticspecific epsin deficiency promotes
VEGFC-induced lymphangiogenesis under diabetic conditions, Chen and colleagues performed mouse corneal micropocket assay, which is a method of assessing angiogenesis. "By using the cornea micropocket assay, we demonstrated that epsin deficiency significantly improved lymphatic sprouting and increased lymphatic vessel branching in non-diabetic LEC-iDKO and diabetic LEC-iDKO mice," adds Chen.

Next, the authors used a model of lymphoedema that uses mouse tails to study lymphangiogenesis in skin regeneration. By combining this model of lymphoedema with intravital multiphoton microscopy, Chen and colleagues discovered that lymphatic-specific loss of epsins reversed the functional deficit in lymphatic function observed with hyperglycaemia and led to recovery from tail oedema.

"In our study, we were also able to show that epsins 1 and 2 were significantly upregulated in patients with diabetes mellitus compared with healthy individuals," concludes Chen. "Therefore, our findings suggest that an epsin-dependent mechanism is involved in impaired lymphangiogenesis in humans and highlight a potential therapeutic target for improving recovery of wound healing in patients with T2DM."

Alan Morris

ORIGINAL ARTICLE Wu, H. et al. Epsin deficiency promotes lymphangiogenesis through regulation of VEGFR3 degradation in diabetes. J. Clin. Invest. https://doi.org/10.1172/JCl96063 (2018) 